\title{
Effective Floquet Hamiltonian for spin $I=1$ in magic angle spinning NMR using contact transformation
}

\author{
MANOJ KUMAR PANDEY and MANGALA SUNDER KRISHNAN* \\ Department of Chemistry, Indian Institute of Technology - Madras, Chennai 600036 \\ e-mail: mangal@iitm.ac.in; mangala_sunderk@yahoo.com
}

MS received 2 July 2007; accepted 22 August 2007

\begin{abstract}
Contact transformation is an operator transformation method in time-independent perturbation theory which is used successfully in molecular spectroscopy to obtain an effective Hamiltonian. Floquet theory is used to transform the periodic time-dependent Hamiltonian, to a time-independent Floquet Hamiltonian. In this article contact transformation method has been used to get the analytical representation of Floquet Hamiltonian for quadrupolar nuclei with spin $I=1$ in the presence of an RF field and first order quadrupolar interaction in magic angle spinning NMR experiments. The eigenvalues of contact transformed Hamiltonian as well as Floquet Hamiltonian have been calculated and a comparison is made between the eigenvalues obtained using the two Hamiltonians.
\end{abstract}

Keywords. Floquet Hamiltonians; contact transformations in NMR; Spin-1 MAS NMR; effective Hamiltonians.

\section{Introduction}

Solid state nuclear magnetic resonance spectroscopy is an important technique to study structures, dynamics and electric charge distribution around nuclei in solids. It is also more difficult to perform and analyse than solution state NMR because in solutions rapid tumbling of the molecules leads to averaging out of anisotropic NMR interactions such as chemical shift, dipole-dipole interactions and quadrupolar interactions resulting in narrow spectral lines in the latter case. However, in solid state, the very same interactions can lead to broadening of the spectral lines. ${ }^{1,2}$

The study of nuclear electric quadrupolar interactions by solid state NMR spectroscopic technique is important in the analysis of electric charge distributions and is also relevant as almost three-fourths of NMR active nuclei in periodic table are quadrupolar in nature. Quadrupolar nuclei $(I>1 / 2)$ have nonspherical distribution of charges inside the nucleus and thus possess a nuclear quadrupole moment which interacts with the electric field gradient present at the nucleus developed by surrounding charges outside the nucleus (largely due to electrons and thus contributing to the chemistry of such solids) and results in

\footnotetext{
*For correspondence
}

electrostatic energy. This interaction energy ranges from a few $\mathrm{KHz}$ to several $\mathrm{MHz}$ in frequency units and is usually stronger than other anisotropic nuclear spin interactions such as chemical shift and dipolar couplings, causing substantial broadening of spectral line shapes for powder samples.

The most common line narrowing technique in solid state NMR spectroscopy is magic angle spinning (MAS) developed by Andrew et $a l^{3}$ and Lowe et $a l^{4}$ which averages out non-selectively all kinds of first order anisotropic interactions present in the system. MAS involves rotation of samples about an axis making an angle of $54.736^{\circ}$ with respect to the applied static magnetic field at specific spinning speeds and imparts a periodic time-dependence to the spin Hamiltonian which renders theoretical description of the experiments difficult. Time-dependent, periodic Hamiltonians have been studied either by the average Hamiltonian theory of John Waugh and coworkers $^{5,6}$ or by Floquet theory by Vega and coworkers ${ }^{7-13}$ based on the approach by Shirley ${ }^{14}$ in 1965 . In this paper we make use of the latter approach and present modifications to Floquet Hamiltonians derived earlier. The transformation of the time-dependent, periodic Hamiltonian to a time independent Floquet Hamiltonian is a rather straightforward process, however, the basis in which the Floquet Hamiltonian is expressed is an infinite basis set as opposed to the 
finite basis set corresponding to the spin-only basis functions used in NMR. The basis set for Floquet transformation is constructed by the direct product of the spin basis with the Fourier basis. The infinite dimensionality of the basis set is due to the fact that the Fourier index n, can take all integer values ranging from $-\infty$ to $+\infty$ in principle. In practice it is required to truncate this at a suitable value of the Fourier index.

In order to study the dynamics and time evolution of the spin system, it is important to diagonalize the Floquet Hamiltonian (described in the infinite dimensional basis set). In the past these were attempted using numerical diagonalization procedure extensively. An analytic representation of effective Floquet Hamiltonians has been given using the method of contact transformation ${ }^{15}$ or the van Vleck method recently. It has been used to study the MAS NMR of spin systems. ${ }^{16-19}$

In this paper the contact transformation method is extended to obtain an analytic representation of Floquet Hamiltonian for quadrupolar nuclei with spin $I=1$. The focus is on the study of first order quadrupolar interaction in MAS experiments. The extension of the algebra described here to second order quadrupolar interactions is straightforward and is not included here for the sake of brevity. We want to limit ourselves to describing the extension of spin $1 / 2$ algebra to higher spins using spherical tensor operators which are the natural extensions of spin $1 / 2$ angular momentum operators. Spin 1 is the first non-trivial system for this purpose and the methods described in sections $2-4$ can be extended to higher spins as well.

\section{The method of contact transformation}

The method of contact transformation is an operator transformation which involves a series of unitary transformations on a Hamiltonian to obtain an effective Hamiltonian. The unitary transformations are performed sequentially to identify combinations of off-diagonal operators which will have diagonal contributions to the leading diagonal operators. Such combinations lead to eigenvalues closer to the eigenvalues of the overall Hamiltonian and are known as effective Hamiltonians. The advantage of this method lies in the fact that all perturbation corrections are obtained in terms of operators, and hence can be used as effective Hamiltonians for the study of nuclear spin dynamics.
The general form of the Hamiltonian expressed in terms of a series of terms of decreasing order of magnitude is given by

$$
H=H_{0}+\lambda H_{1}+\lambda^{2} H_{2}+\ldots
$$

where $\lambda$ is the perturbation parameter. A series of unitary transformations represented collectively by $U$ on the Hamiltonian $H$ is performed as

$$
\begin{aligned}
\tilde{H}= & U H U^{-1}=\ldots e^{\left(i \lambda^{n} S_{n}\right)} \ldots e^{\left(i \lambda^{2} S_{2}\right)} \\
& \times e^{\left(i \lambda S_{1}\right)} H e^{\left(-i \lambda S_{1}\right)} e^{\left(-i \lambda^{2} S_{2}\right)} \ldots . . e^{\left(-i \lambda^{n} S_{n}\right)} \ldots
\end{aligned}
$$

in which the exponential operators represent contact transformation operators (themselves being unitary). $S_{n}$ 's in each exponential are chosen as Hermitian operators in order to make the exponential operators unitary.

The transformed Hamiltonian $H^{(1)}$ after the first transformation is given by,

$$
\begin{aligned}
H^{(1)} & =U_{1} H U_{1}^{-1}=\mathrm{e}^{\left(i \lambda S_{1}\right)} H \mathrm{e}^{\left(-i \lambda S_{1}\right)} \\
& =H_{0}^{(1)}+\lambda H_{1}^{(1)}+\lambda^{2} H_{2}^{(1)}+\ldots .
\end{aligned}
$$

The importance of this method lies in the determination of $S$ operators. $S_{1}$ is chosen in such a way that $H_{1}^{(1)}$ has no off-diagonal elements up to order $\lambda$.

On expanding $H^{(1)}$ we have,

$$
\begin{aligned}
H^{(1)}= & H_{0}+\lambda\left(H_{1}+i\left[S_{1}, H_{0}\right]\right) \\
& +\lambda^{2}\left(H_{2}+i\left[S_{1}, H_{1}\right]-\frac{1}{2}\left[S_{1},\left[S_{1}, H_{0}\right]\right]\right)+\ldots \\
& =H_{0}^{(1)}+\lambda H_{1}^{(1)}+\lambda^{2} H_{2}^{(1)}+\ldots
\end{aligned}
$$

By equating powers of $\lambda$ on both sides we get,

$$
\begin{aligned}
& H_{0}^{(1)}=H_{0} \\
& H_{1}^{(1)}=H_{1}+i\left[S_{1}, H_{0}\right] \\
& H_{2}^{(1)}=H_{2}+i\left[S_{1}, H_{1}\right]-\frac{1}{2}\left[S_{1},\left[S_{1}, H_{0}\right]\right]
\end{aligned}
$$

The diagonal part of the above Hamiltonian gives diagonal correction of order $\lambda^{2}$ to the zeroth order Hamiltonian in addition to the diagonal term of $\mathrm{H}_{2}$. Thus the Hamiltonian $H_{0}^{(1)}+\lambda H_{1}^{(1) d}+\lambda^{2} H_{2}^{(1) d}$ is more effective than the Hamiltonian $H_{0}+\lambda H_{1}^{d}+\lambda^{2} H_{2}^{d}$.

The general term $H_{n}^{(1)}$ is given by, 


$$
\left.H_{n}^{(1)}=H_{n}+\sum_{m=0}^{n-1} \frac{i^{n-m}}{(n-m) !}[\underbrace{S_{1},\left[S_{1}, \ldots \ldots\right.}_{n-m}\left[S_{1}, H_{m}\right] \ldots]\right]
$$

The above procedure is repeated to perform second or higher order transformations on $H^{(1)}$.

\section{Floquet Hamiltonian for spin $I=1$}

The time-dependent MAS Hamiltonian ${ }^{20}$ for a spin $I=1$, exhibiting a first order quadrupolar interaction and experiencing an RF field of strength $\omega_{1 I}$ is given as,

$$
\begin{array}{r}
H_{I}(t)=-\omega_{1 I} I_{X}+\omega_{Q}\left[g_{1} \cos \left(\omega_{r} t+\psi_{1}\right)+\right. \\
\left.g_{2} \cos \left(2 \omega_{r} t+\psi_{2}\right)\right]\left[3 I_{z}^{2}-I^{2}\right]
\end{array}
$$

where, $\omega_{Q}$ is the quadrupolar coupling frequency, $\omega_{r}$ is the rotor frequency, $\eta$ is the asymmetry parameter and

$$
\begin{gathered}
g_{1}=-\frac{1}{2} \sin 2 \theta_{m} \sin \beta_{I}\left[\left(3+\eta \cos 2 \gamma_{I}\right)^{2}\right. \\
\left.\cos ^{2} \beta_{I}+\eta^{2} \sin ^{2} 2 \gamma_{I}\right]^{1 / 2} \\
g_{2}=\frac{1}{2} \sin ^{2} \theta_{m}\left[\left\{\frac{3}{2} \sin ^{2} \beta_{I}-\frac{\eta}{2} \cos 2 \gamma_{I}\left(1+\cos ^{2} \beta_{I}\right)\right\}^{2}\right. \\
\left.+\eta^{2} \cos ^{2} \beta_{I} \sin ^{2} 2 \gamma_{I}\right]^{1 / 2} \\
\psi_{1}=\alpha_{I}+\tan ^{-1}\left[\eta \frac{\sin 2 \gamma_{I}}{\left(3+\eta \cos 2 \gamma_{I}\right) \cos \beta_{I}}\right] \\
\psi_{2}=2 \alpha_{I}+\tan ^{-1}\left[-\eta \frac{\cos \beta_{I} \sin 2 \gamma_{I}}{\frac{3}{2} \sin ^{2} \beta_{I}-\frac{\eta}{2} \cos 2 \gamma_{I}\left(1+\cos ^{2} \beta_{I}\right)}\right] .
\end{gathered}
$$

The transformation of this periodic time-dependent Hamiltonian to Floquet Hamiltonian is done using standard procedure described elsewhere. ${ }^{10}$ The corresponding Floquet Hamiltonian is given by,

$$
\begin{aligned}
H_{I}^{F}= & \omega_{r}\left[N^{11}+N^{22}+N^{33}\right] \\
& -\frac{\omega_{1 I}}{\sqrt{2}}\left[\left(I_{+}\right)_{0}^{12}+\left(I_{-}\right)_{0}^{21}+\left(I_{+}\right)_{0}^{23}+\left(I_{-}\right)_{0}^{32}\right] \\
& +2 \sum_{\substack{n=-2 \\
n \neq 0}}^{2} \omega_{n}^{Q}\left[Z_{n}^{11}-2 Z_{n}^{22}+Z_{n}^{33}\right]
\end{aligned}
$$

where,

$$
\begin{aligned}
& 1 \equiv|+1\rangle, 2 \equiv|0\rangle, 3 \equiv|-1\rangle \text { and } \\
& \omega_{n}^{Q}=\frac{1}{2} \omega_{Q} g_{|n|} \exp \left\{i \frac{n}{|n|} \psi_{|n|}\right\} .
\end{aligned}
$$

The matrix elements of the complete set of Floquet operators for spin $I=1$ are given in a basis set (which is infinite dimensional) and a spin basis set (which is three-dimensional), i.e. $|n\rangle \otimes|\operatorname{Im}\rangle(n=-\infty, \ldots,-1,0$, $1, \ldots \infty, m=-1,0,1$ ) as

$$
\begin{aligned}
& \left\langle 1, n\left|N^{11}\right| 1, n\right\rangle=n,\left\langle 2, n\left|N^{22}\right| 2, n\right\rangle=n, \\
& \left\langle 3, n\left|N^{33}\right| 3, n\right\rangle=n,\left\langle 1, n\left|Z_{m}^{11}\right| 1, n+m\right\rangle=\frac{1}{2},
\end{aligned}
$$

$\left\langle 2, n\left|Z_{m}^{22}\right| 2, n+m\right\rangle=\frac{1}{2},\left\langle 3, n\left|Z_{m}^{33}\right| 3, n+m\right\rangle=\frac{1}{2}$,

$\left\langle 1, n\left|\left(I_{+}\right)_{m}^{12}\right| 2, n+m\right\rangle=1,\left\langle 2, n\left|\left(I_{+}\right)_{m}^{23}\right| 3, n+m\right\rangle=1$,

$\left\langle 2, n\left|\left(I_{-}\right)_{m}^{21}\right| 1, n+m\right\rangle=1,\left\langle 3, n\left|\left(I_{-}\right)_{m}^{32}\right| 2, n+m\right\rangle=1$,

$\left\langle 1, n\left|\left(Z_{+}\right)_{m}^{12}\right| 2, n+m\right\rangle=\frac{1}{2},\left\langle 2, n\left|\left(Z_{+}\right)_{m}^{23}\right| 3, n+m\right\rangle=-\frac{1}{2}$,

$\left\langle 2, n\left|\left(Z_{-}\right)_{m}^{21}\right| 1, n+m\right\rangle=\frac{1}{2},\left\langle 3, n\left|\left(Z_{-}\right)_{m}^{32}\right| 2, n+m\right\rangle=-\frac{1}{2}$,

$\left\langle 1, n\left|\left(I_{+}^{2}\right)_{m}^{13}\right| 3, n+m\right\rangle=1,\left\langle 3, n\left|\left(I_{-}^{2}\right)_{m}^{31}\right| 1, n+m\right\rangle=1$.

\section{Application of contact transformation}

The Floquet Hamiltonian in (8) is decomposed into zero order and first order perturbing Hamiltonians as,

$$
\begin{aligned}
H_{0}= & \omega_{r}\left[N^{11}+N^{22}+N^{33}\right]- \\
& \frac{\omega_{1 I}}{\sqrt{2}}\left[\left(I_{+}\right)_{0}^{12}+\left(I_{-}\right)_{0}^{21}+\left(I_{+}\right)_{0}^{23}+\left(I_{-}\right)_{0}^{32}\right] \\
H_{1}= & 2 \sum_{\substack{n=-2 \\
n \neq 0}}^{2} \omega_{n}^{Q}\left[Z_{n}^{11}-2 Z_{n}^{22}+Z_{n}^{33}\right] .
\end{aligned}
$$

The transformation function $S_{1}$ is chosen such that the commutator $i\left[S_{1}, H_{0}\right]$ compensates off-diagonal terms present in $H_{1}$. In order to solve for $S_{1}$, it is 
written as a linear combination of complete set of operators for spin $I=1$ system with arbitrary coefficients as follows,

$$
S_{1}=i\left[\sum_{\substack{n=2 \\
n \neq 0}}^{-2}\left\{\begin{array}{l}
A_{n}\left(Z_{n}^{11}-Z_{n}^{33}\right)+B_{n}\left\{\left(I_{+}\right)_{n}^{12}+\left(I_{+}\right)_{n}^{23}\right\}+ \\
C_{n}\left\{\left(I_{-}\right)_{n}^{21}+\left(I_{-}\right)_{n}^{32}\right\}+ \\
D_{n}\left(Z_{n}^{11}-2 Z_{n}^{22}+Z_{n}^{33}\right)+E_{n}\left\{\left(Z_{+}\right)_{n}^{12}+\right. \\
\left.\left(Z_{+}\right)_{n}^{23}\right\}+F_{n}\left\{\left(Z_{-}\right)_{n}^{21}+\left(Z_{-}\right)_{n}^{32}\right\} \\
+G_{n}\left\{\left(I_{+}^{2}\right)_{n}^{13}\right\}+H_{n}\left\{\left(I_{-}^{2}\right)_{n}^{31}\right\}
\end{array}\right\} .\right.
$$

The coefficients are obtained by solving the equation,

$$
H_{1}+i\left[S_{1}, H_{0}\right]=0 .
$$

Expressions for coefficients $A_{n}, B_{n}, C_{n}, D_{n}, E_{n}, F_{n}$, $G_{n}$ and $H_{n}$ are obtained as

$$
D_{n}=\frac{2 \omega_{n}^{Q}\left(n \omega_{r}-\omega_{1 I}\right)\left(n \omega_{r}+\omega_{1 I}\right)}{n^{3} \omega_{r}^{3}-4 n \omega_{r} \omega_{1 I}^{2}}
$$

$$
\begin{aligned}
& E_{n}=-F_{n}=\frac{3 \sqrt{2} \omega_{n}^{Q} \omega_{1 I}}{n^{2} \omega_{r}^{2}-4 \omega_{1 I}^{2}}, \\
& G_{n}=H_{n}=\frac{3 \omega_{n}^{Q} \omega_{1 I}}{n^{3} \omega_{r}^{3}-4 n \omega_{r} \omega_{1 I}^{2}} .
\end{aligned}
$$

The coefficients $A_{n}, B_{n}$ and $C_{n}$ are identically zero for all $n$.

Using the expression for $S_{1}$, diagonal corrections to the Hamiltonian are obtained from

$$
H_{2}^{(1)}=H_{2}+i\left[S_{1}, H_{1}\right]-\frac{1}{2}\left[S_{1},\left[S_{1}, H_{0}\right]\right] .
$$

In the present case $H_{2}=0$ and $H_{1}$ is off-diagonal, so we have

$$
H_{2}^{(1)}=\frac{i}{2}\left[S_{1}, H_{1}\right] .
$$

The commutation relations used in (12) and (13) are given in table 1 . Using these relations the diagonal

Table 1. Commutation relations derived for various Floquet operators for the case of spin $I=1$ system.

$$
\begin{aligned}
& {\left[Z_{n}^{11}, N^{11}\right]=n Z_{n}^{11}\left[Z_{n}^{22}, N^{22}\right]=n Z_{n}^{22} \quad\left[Z_{n}^{33}, N^{33}\right]=n Z_{n}^{33}} \\
& {\left[Z_{n}^{11},\left(I_{+}\right)_{m}^{12}\right]=\frac{1}{2}\left(I_{+}\right)_{n+m}^{12} \quad\left[Z_{n}^{22},\left(I_{+}\right)_{m}^{12}\right]=-\frac{1}{2}\left(I_{+}\right)_{n+m}^{12} \quad\left[Z_{n}^{33},\left(I_{+}\right)_{m}^{12}\right]=0} \\
& {\left[Z_{n}^{11},\left(I_{-}\right)_{m}^{21}\right]=-\frac{1}{2}\left(I_{-}\right)_{n+m}^{21} \quad\left[Z_{n}^{22},\left(I_{-}\right)_{m}^{21}\right]=\frac{1}{2}\left(I_{-}\right)_{n+m}^{21} \quad\left[Z_{n}^{33},\left(I_{-}\right)_{m}^{21}\right]=0} \\
& {\left[Z_{n}^{11},\left(I_{+}\right)_{m}^{23}\right]=0 \quad\left[Z_{n}^{22},\left(I_{+}\right)_{m}^{23}\right]=\frac{1}{2}\left(I_{+}\right)_{n+m}^{23} \quad\left[Z_{n}^{33},\left(I_{+}\right)_{m}^{23}\right]=-\frac{1}{2}\left(I_{+}\right)_{n+m}^{12}} \\
& {\left[Z_{n}^{11},\left(I_{-}\right)_{m}^{32}\right]=0 \quad\left[Z_{n}^{22},\left(I_{-}\right)_{m}^{32}\right]=-\frac{1}{2}\left(I_{-}\right)_{n+m}^{21} \quad\left[Z_{n}^{33},\left(I_{-}\right)_{m}^{32}\right]=\frac{1}{2}\left(I_{-}\right)_{n+m}^{21}} \\
& {\left[Z_{n}^{11},\left(I_{+}^{2}\right)_{m}^{13}\right]=\frac{1}{2}\left(I_{+}^{2}\right)_{n+m}^{13} \quad\left[Z_{n}^{22},\left(I_{+}^{2}\right)_{m}^{13}\right]=0 \quad\left[Z_{n}^{33},\left(I_{+}^{2}\right)_{m}^{13}\right]=-\frac{1}{2}\left(I_{+}^{2}\right)_{n+m}^{13}} \\
& {\left[Z_{n}^{11},\left(I_{-}^{2}\right)_{m}^{31}\right]=-\frac{1}{2}\left(I_{-}^{2}\right)_{n+m}^{31} \quad\left[Z_{n}^{22},\left(I_{+}^{2}\right)_{m}^{13}\right]=0 \quad\left[Z_{n}^{33},\left(I_{+}^{2}\right)_{m}^{31}\right]=\frac{1}{2}\left(I_{-}^{2}\right)_{n+m}^{31}} \\
& {\left[\left(I_{+}\right)_{n}^{12},\left(N^{11}+N^{22}\right)\right]=n\left(I_{+}\right)_{n}^{12} \quad\left[\left(I_{-}\right)_{n}^{21},\left(N^{11}+N^{22}\right)\right]=n\left(I_{-}\right)_{n}^{21}} \\
& {\left[\left(I_{+}\right)_{n}^{23},\left(N^{22}+N^{33}\right)\right]=n\left(I_{+}\right)_{n}^{23} \quad\left[\left(I_{-}\right)_{n}^{32},\left(N^{22}+N^{33}\right)\right]=n\left(I_{-}\right)_{n}^{32}} \\
& {\left[\left(I_{+}^{2}\right)_{n}^{13},\left(N^{11}+N^{33}\right)\right]=n\left(I_{+}^{2}\right)_{m}^{13} \quad\left[\left(I_{-}^{2}\right)_{n}^{31},\left(N^{11}+N^{33}\right)\right]=n\left(I_{-}^{2}\right)_{m}^{31}} \\
& {\left[\left(I_{+}\right)_{n}^{23},\left(I_{-}\right)_{m}^{32}\right]=2\left(Z_{n+m}^{22}-Z_{n+m}^{33}\right) \quad\left[\left(I_{+}\right)_{n}^{12},\left(I_{-}\right)_{m}^{21}\right]=2\left(Z_{n+m}^{11}-Z_{n+m}^{22}\right)} \\
& {\left[\left(I_{+}\right)_{n}^{12},\left(I_{+}\right)_{m}^{23}\right]=\left(I_{+}^{2}\right)_{n+m}^{13} \quad\left[\left(I_{-}\right)_{n}^{21},\left(I_{-}\right)_{m}^{32}\right]=-\left(I_{-}^{2}\right)_{n+m}^{31} \quad\left[\left(I_{+}\right)_{n}^{12},\left(I_{-}^{2}\right)_{m}^{31}\right]=-\left(I_{-}\right)_{n+m}^{32}} \\
& {\left[\left(I_{+}\right)_{n}^{23},\left(I_{-}^{2}\right)_{m}^{31}\right]=\left(I_{-}\right)_{n+m}^{21}\left[\left(I_{-}\right)_{n}^{32},\left(I_{+}^{2}\right)_{m}^{13}\right]=-\left(I_{+}\right)_{n+m}^{12} \quad\left[\left(I_{-}\right)_{n}^{21},\left(I_{+}^{2}\right)_{m}^{13}\right]=\left(I_{+}\right)_{n+m}^{23}} \\
& {\left[\left(I_{+}\right)_{n}^{12},\left(I_{-}\right)_{m}^{32}\right]=0 \quad\left[\left(I_{+}\right)_{n}^{23},\left(I_{-}\right)_{m}^{21}\right]=0 \quad\left[\left(I_{-}\right)_{n}^{21},\left(I_{+}\right)_{m}^{23}\right]=0} \\
& {\left[\left(I_{+}\right)_{n}^{12},\left(I_{+}^{2}\right)_{m}^{13}\right]=0 \quad\left[\left(I_{+}\right)_{n}^{23},\left(I_{+}^{2}\right)_{m}^{13}\right]=0 \quad\left[\left(I_{-}\right)_{n}^{32},\left(I_{-}^{2}\right)_{m}^{31}\right]=0} \\
& {\left[\left(I_{-}\right)_{n}^{21},\left(I_{-}^{2}\right)_{m}^{31}\right]=0 \quad\left[\left(I_{+}^{2}\right)_{n}^{13},\left(I_{-}^{2}\right)_{m}^{31}\right]=2\left(Z_{n+m}^{11}-Z_{n+m}^{33}\right)}
\end{aligned}
$$


Table 2. Parameters used for the calculation of eigenvalues.

\begin{tabular}{lc}
\hline$\theta_{m}$ & $54 \cdot 736^{\circ}$ \\
$\beta_{1}$ & $0^{\circ}$ \\
$\alpha_{I}$ & $90^{\circ}$ \\
$\gamma_{I}$ & $60^{\circ}$ \\
$\eta$ & $0 \cdot 1$ \\
$\omega_{1 I}$ & $44 \mathrm{KHz}$ \\
$\omega_{Q}$ & $80 \mathrm{KHz}$ \\
\hline
\end{tabular}

Table 3. Comparison between eigenvalues of transformed and untransformed Floquet Hamiltonian.

\begin{tabular}{lcc}
\hline$\omega_{r}$ & $\begin{array}{c}\text { First } \\
\text { transformation }\end{array}$ & $\begin{array}{c}\text { Numerical } \\
\text { diagonalization }\end{array}$ \\
\hline $10 \mathrm{kHz}$ & $-4 \cdot 14387$ & $-4 \cdot 14349$ \\
& $0 \cdot 0$ & $0 \cdot 0$ \\
& $4 \cdot 14387$ & $4 \cdot 14349$ \\
$20 \mathrm{kHz}$ & $-4 \cdot 17196$ & $-4 \cdot 17129$ \\
& $0 \cdot 0$ & $0 \cdot 0$ \\
$30 \mathrm{kHz}$ & $4 \cdot 17196$ & $4 \cdot 17129$ \\
& $-14 \cdot 25496$ & $-14 \cdot 25261$ \\
& $0 \cdot 0$ & $0 \cdot 0$ \\
& $14 \cdot 25496$ & $14 \cdot 25261$ \\
\hline
\end{tabular}

corrections obtained for zero order Hamiltonian is given by

$$
H_{2}^{(1)}=\left[\begin{array}{l}
\frac{3}{4} \sum_{n=-2}^{2}\left\{\left(E_{n} \omega_{-n}^{Q}\right)\left\{\left(I_{+}\right)_{0}^{12}+\left(I_{+}\right)_{0}^{23}\right\}\right. \\
\left.-\left(F_{n} \omega_{-n}^{Q}\right)\left\{\left(I_{-}\right)_{0}^{21}+\left(I_{-}\right)_{0}^{32}\right\}\right\}
\end{array}\right]
$$

The final form for the diagonal part of the oncetransformed equation, truncated to one order is given as

$$
\begin{aligned}
H_{\text {eff }}= & \omega_{r}\left[N^{11}+N^{22}+N^{33}\right]+ \\
& \sum_{\substack{n=-2 \\
n \neq 0}}^{2}\left[\begin{array}{l}
\left\{\frac{3}{4}\left(E_{n} \omega_{-n}^{Q}\right)-\frac{\omega_{1 I}}{\sqrt{2}}\right\}\left\{\left(I_{+}\right)_{0}^{12}+\left(I_{+}\right)_{0}^{23}\right\} \\
-\left\{\frac{3}{4}\left(F_{n} \omega_{-n}^{Q}\right)+\frac{\omega_{1 I}}{\sqrt{2}}\right\}\left\{\left(I_{-}\right)_{0}^{21}+\left(I_{-}\right)_{0}^{32}\right\}
\end{array}\right] .
\end{aligned}
$$

\section{Numerical results}

In this section, the eigenvalues of the Hamiltonian in (16) obtained by contact transformation method are compared with the untransformed Floquet Hamiltonian given by (9) and (10). A matrix of dimension $213 \times 213$ was employed for the diagonalization of untransformed Hamiltonian ( $n=35$, dimension is $(2 n+1) \times 3)$, while in the case of contact transformation effective Hamiltonian corresponding to a given Fourier label $n$ is diagonalized. The latter is a $3 \times 3$ matrix for a spin- 1 system. The values given here are for $n=0$. Due to the periodicity of the untransformed Hamiltonian, the eigenvalues for any $n$ can be generated from the eigenvalues of the case for $n=0$. All eigenvalues for a given $n$ are obtained using the equation $E_{i}^{n}=E_{i}^{0}+n \omega_{r}$.

From table 3 it is clear that the eigenvalues obtained from contact transformation are in good agreement with the eigenvalues obtained for untransformed Floquet Hamiltonian using a much bigger basis set. However, the agreement is less striking and quite far off for values of beta significantly different from zero. In table 4 eigenvalues for two non-zero beta values for which the first order correction does not give satisfactory results are given. This is our current topic of interest.

The application of RF pulse to a spin- 1 system is shown here as a preliminary result. The approach has to be generalized in order to include free evolution of the spin systems between RF pulses. Several experimental pulse schemes are proposed to be studied using this approach for both spin- 1 and spin-3/2 systems which are most commonly used quadrupolar nuclear spins in this area.

\section{Conclusion}

In this article, a procedure has been evolved for generalizing the contact transformation method developed for the analysis of Floquet Hamiltonians to dipolar coupled spin $1 / 2$ systems to the study of quadrupolar spins with $I=1$ as an example. The model is developed for first order interactions though it is equally applicable for second order quadrupolar interactions. The commutators given in table 1 form the basis for the application of contact transformation method. Only preliminary results have been reported here and no attempt has been made to include spin 1 experimental results. The extension to spin 1 using spherical tensor operators is a novel approach 
Table 4. Comparison between eigenvalues of transformed and untransformed Floquet Hamiltonian for two non-zero $\beta_{I}$ values with retaining all other parameters of table 2 .

\begin{tabular}{lcccc}
\hline & $\begin{array}{c}\text { First } \\
\text { transformation } \\
\left(\beta_{I}=90^{\circ}\right)\end{array}$ & $\begin{array}{c}\text { Numerical } \\
\text { diagonalization } \\
\left(\beta_{I}=90^{\circ}\right)\end{array}$ & $\begin{array}{c}\text { First } \\
\text { transformation } \\
\left(\beta_{I}=45^{\circ}\right)\end{array}$ & $\begin{array}{c}\text { Numerical } \\
\text { diagonalization } \\
\left(\beta_{I}=45^{\circ}\right)\end{array}$ \\
\hline $10 \mathrm{kHz}$ & $-2 \cdot 40156$ & $-2 \cdot 10225$ & $-3 \cdot 10701$ & $-4 \cdot 60134$ \\
& $0 \cdot 0$ & $0 \cdot 0$ & $0 \cdot 0$ & $0 \cdot 0$ \\
$20 \mathrm{kHz}$ & $2 \cdot 40156$ & $2 \cdot 10225$ & $3 \cdot 10701$ & $4 \cdot 60134$ \\
& $-4 \cdot 13931$ & $-8 \cdot 47241$ & $-3 \cdot 49869$ & $-3 \cdot 80512$ \\
$30 \mathrm{kHz}$ & $0 \cdot 0$ & $0 \cdot 0$ & $0 \cdot 0$ & $0 \cdot 0$ \\
& $4 \cdot 13931$ & $8 \cdot 47241$ & $3 \cdot 49869$ & $3 \cdot 80512$ \\
& $-13 \cdot 45274$ & $-10 \cdot 79703$ & $-14 \cdot 73702$ & $-10 \cdot 60056$ \\
& $0 \cdot 0$ & $0 \cdot 0$ & $0 \cdot 0$ & $0 \cdot 0$ \\
& $13 \cdot 45274$ & $10 \cdot 79703$ & $14 \cdot 73702$ & $10 \cdot 60056$ \\
\hline
\end{tabular}

which we hope to pursue further for more elaborate studies on quadrupolar systems of $I=3 / 2$ and higher.

\section{Acknowledgments}

M K P thanks IIT Madras for a research fellowship which enabled him to undertake this work. This research was supported by IIT Madras research funds.

\section{References}

1. Mehring M 1983 Principles of high resolution NMR in solids (Berlin: Springer)

2. Haeberlen U 1976 Principles of high resolution NMR in solids: Selective averaging (New York: Academic) suppl. 1

3. Andrew E R, Bradbury A and Eades R G 1958 Nature (London) 182 1659; Andrew E R, Bradbury A and Eades R G 1959 Nature (London) 1831802

4. Lowe I J 1959 Phys. Rev. Lett. 2285

5. Maricq M M and Waugh J S 1979 J. Chem. Phys. 70 3300

6. Maricq M M 1982 Phys. Rev. B25 6622
7. Schmidt A and Vega S 1987 J. Chem. Phys 876895

8. Schmidt A and Vega S 1992 J. Chem. Phys. 962655

9. Weintraub O and Vega S 1993 J. Magn. Reson. (Series A) 105245

10. Vega S 1996 In Encyclopedia of nuclear magnetic resonance (New York: Wiley) vol 3, p 2011; and references therein

11. Boender G, Vega S and De Groot H J M 1998 Mol. Phys. 95921

12. Levante T O, Baldus M, Meier B H and Ernst 1995 Mol. Phys. 861195

13. Filip C, Filip X, Demcos D E and Hafner S 1997 Mol. Phys. 92757

14. Shirley J H 1965 Phys. Rev. B138 979

15. Papousek D and Aliev M R 1982 Molecular vibrational-rotational spectra (Amsterdam: Elsevier)

16. Ramesh R and Krishnan M S 2001 J. Chem. Phys. 1145967

17. Ramesh R and Griffin R G 2005 J. Chem. Phys. 122 164502

18. Ramesh R, Bajaj V S and Griffin R G 2005 J. Chem. Phys. 122164503

19. Ramesh R, Lewandowski J R, van der Wel P C A and Griffin R G 2006 J. Chem. Phys. 124214107

20. Marks D, Zumbulyadis N and Vega S 1996 J. Magn. Reson. (Series A) 12216 